\title{
Encapsulation - A Perspective Improving Functional Properties of Flour-Based Confectionary
}

\author{
Sanja Oručević Žuljević* and Asima Akagić \\ Faculty of Agriculture and Food Sciences, Department of Food Technology, University of Sarajevo, Bosnia and Herzegovina
}

*Corresponding author: Sanja Oručević Žuljević, Faculty of Agriculture and Food Sciences, Department of Food Technology, University of Sarajevo, Zmaja od Bosne 871000 Sarajevo, Bosnia and Herzegovina.

To Cite This Article: Sanja Oručević Žuljević, Asima Akagić. Encapsulation - A Perspective Improving Functional Properties of Flour-Based Confectionary. 2020 - 9(4). AJBSR.MS.ID.001405. DOI: 10.34297/AJBSR.2020.09.001405.

Received: 製 May 13, 2020; Published: 盋 July 08, 2020

\section{Introduction}

Flour-based confectionary is the most popular confectionary and have an important position in food technology in general. Those products present the most often consumed confectionary products and their growth in EU market has been increasing per capita consumption [1] owing to their sweet taste and nice aroma and flavor, easy accessibility-ready to use and well-established habits [2], lower production costs, convenience and long shelf life [3].

Due to consumers demands for the health food the improvements of this type of confectionary are becoming more and more frequent. Biscuits together with chocolate products are on the first place with $8.7 \%$ of total European food innovation [1].

By adding different functional components, it is possible to make flour-based confectionary are with functional features offer health benefits that extend beyond their nutritional value by e.g. incorporating inulin and acacia gum [3]; chickpea and rice bran as a source of dietary fiber and active healthy components to make functional biscuits for lowering blood lipids [4]; addition of the prebiotic ingredient fructo-oligosaccharide found to provide both nutritional and technological advantages in biscuits [5].

The functional foods include foods containing minerals, vitamins, fatty acids, dietary fiber, foods with the addition of biologically active substances such as antioxidants and probiotics [6]. According to Jain [7] functional food ingredients have been brought to the consumers mainly through dairy or confectionery products [7].

Berries such (strawberries, cranberries, blueberries, raspberries and blackberries) in general may offer health promoting benefits and present excellent choice to improve flour-based confectionary from functional aspect, as they are low in calories, and abundant in anthocyanins and polyphenolic components and have a good potential to encapsulate, as well. The importance of cornelian cherry fruits in the class of berry fruit is especially emphasized [8] and traditional wild fruit (rosehip, cornelian cherry, wild strawberry and bilberry) as an important foodstuff in the local population nutrition which has been recognized in folk medicine since ancient times [9-11].

\section{Encapsulation}

Encapsulation technology is now well developed and accepted within pharmaceutical, chemical, cosmetic, foods and printing industries. In food products, fats and oils, aroma compounds and oleo-resins, vitamins, minerals, colorants, and enzymes could encapsulate while controlling their release and show promising approach to develop functionally active food products.

Microencapsulation describes an even finer degree of encapsulation technology and is one of the earliest examples of applied nanotechnology, which is the science of controlling matter at the atomic and molecular physical level [12]. It defined as a process to entrap active agents within a carrier material (wall material) and presents a useful tool to improve delivery of bioactive molecules and living cells into foods. This technology allows protection of bioactive components by completely enveloping and covering in small capsules that release their contents at controlled rates over prolonged periods and under specific conditions $[13,14]$ and presents a suitable method capable of delivering adequate amounts of viable probiotic bacteria into the gastrointestinal tract $[15,16]$. Co-encapsulation is especially useful as this concept takes into account the synergistic effect of multiple bioactives in enhancing bioactivity to target specific health benefits [17]. 


\section{Applying Encapsulation in Flour-Based Confection-} ary

To produce effective encapsulated products, the choice of coating material and method of microencapsulation process are most important and it also depends on the end use of the product and the processing conditions involved [18]. Lakkis [12] states the spray chilling is the most frequently used processes, due to many benefits such as microcapsule small particle size, good barrier properties, and protection through handling, mixing, and storage of bakery formulations, as well as ability to design the desired mode of release [12]. Nevertheless, other experiences are also presented. Among the available microencapsulation methods, spray drying offers several advantages, it is a continuous process has an economical potential technology and required short residence time $[19,20]$. According to Alvim et al. [21] both microencapsulation methods can be used to obtain microparticles as potential protection vehicles in the application of sensitive substances such as ascorbic acid in baked products [21].

As the biscuits or cookies are widely consumed snacks, they are therefore an ideal food for fortification [22]. Incorporation of microencapsulated vitamins into food matrix contributes to improvement food nutritional value, reduce off-flavors, permit timerelease of nutrients, enhance the stability to high temperature and moisture and reduce each nutrient interaction other ingredients [18].

Wilson \& Shah [23] have been considering encapsulation efficiency of vitamin C from various studies. Many studies have been done on this vitamin with variables optimized to determine the most stable way of microencapsulating vitamin $C$ to give the highest retention possible and to use these applications in food fortification [23].

According to Shrestha et al. [22] microencapsulation is a technique that provides protection to highly susceptible micronutrients including 5-methyltetrahydrofolic acid which is regarded as one of the most bioactive forms of folate. The study investigated its stability in microencapsulated form and changes the physical properties in biscuits baked at different temperatures and times regimes. They found that microcapsule with pectin and alginate ratio of 80:20, prepared by spray drying, gave the highest retention (68.6\%) of the 5-methyltetrahydrofolic acid [22].

In study by Kulthe et al. [24] laboratory prepared microencapsulated $\beta$-carotene by spray drying was added to enrich cookies with maida and pearl millet flour in ratio 60:40. Microencapsulation protected $\beta$-carotene maintained its activity during different process regimes of cookies making. According to obtained results the amount of $4 \%$ of microencapsulated $\beta$-carotene enriched pearl millet-based cookies did not affected the overall quality [24].
Fruits and by-products occurred during processing are valuable source of bioactive components and could encapsulate and successfully incorporate into flour-based confectionary to increase them functional properties.

Blueberry microspheres were prepared by spray drying and freeze drying and were evaluated for their impact on the quality of ice cream and cake. The microencapsulation process is effective in the protecting of phenolics against thermal degradation in cake samples and the ultrasonic nozzle microspheres of extract showed the highest anthocyanin retention (79.35\%) than the other microspheres investigated, in enriched cake samples [19]. Luca et al. [25] demonstrated the characteristics of encapsulated phenolic powders obtained from sour cherry pomace in cake making. Encapsulation improved storage stability of the phenolic compounds and increased the resistance of phenolic compounds to the thermal effects. However, degradation of phenolic compounds during baking was high even if they were encapsulated. This study also demonstrated that addition of small amount of encapsulated phenolic compounds into cake before baking did not affect its quality parameters adversely [25].

Encapsulated extract from waste red grape skin in maltodextrin were evaluated on the antioxidant properties and sensory acceptability of whole wheat cocoa biscuits. Extract addition increased the phenolic content and antioxidant capacity of both dough's and biscuits. The results showed that whole wheat cacao biscuits represent an appropriate food matrix to develop pleasant novel products enriched in phenolic compounds from waste grape skins and likeable for regular biscuits consumers [26]. The research of Elsebaie \& Essa [27] focused on extraction of biological compounds from red onion peels, and its microencapsulation to evaluate cake quality integrated with microencapsulated polyphenols, and polyphenols stability assessment after baking. Polyphenols content of encapsulating incorporated cake was enough to claim for the functionality of polyphenols in the cake [27]. Cocoa hulls represent a by-product of cocoa processing and they are a potential source of polyphenols. However, their low stability to oxidation and thermal degradation limits their practical application. The results obtained by Papillo et al. [28] evidenced the usefulness of the microencapsulation process to improve the stability of polyphenolic compounds and antioxidant properties and application in biscuit making [28].

Research of Rosha etal. [29] demonstrated possibility of applying encapsulate oily solutions of lycopene obtained by spray drying technique in cake production. They presented microencapsulation as an alternative to minimize lycopene instability. In addition, it was observed that the microcapsules were able to release pigment and color the studied food system in a homogenous manner [29].

Some high added value components (essential oils, antioxidative and volatile compounds) have significant properties 
from nutritive and medicinal aspects, and antimicrobial activities which can be exploited in the production of functional foods and enable prolonging the shelf life of the final product while their strong and atypical tastes and smells could be successfully masked by encapsulation [20]. Encapsulated thyme (Thymus vulgaris) oil presented the potential for use as a natural food preservative that can be applied to cakes to promote their shelf lives and avoid synthetic preservatives [30]. Green tea extract was encapsulated within electro sprayed protein microparticles, and the protective ability of both systems on the green tea catechins was assessed. Results showed that microencapsulation did not significantly protect during biscuit processing and emphasized the need of assessing the behavior of microencapsulation systems in real food processing conditions [31].

Encapsulation represents a useful method to prevent the formation of potentially harmful compounds in thermally processed foods such as 5-hydroxymethylfurfural (HMF). The potential of sodium chloride encapsulation to mitigate the Maillard reaction on the HMF formation in cookies was tested. The study included 13 cookies samples prepared with recipes containing free or encapsulated $\mathrm{NaCl}$. Increasing $\mathrm{NaCl}$ concentration from 0 to $0.65 \%$ increases HMF concentration up to $75 \%$, whereas in the presence of encapsulated $\mathrm{NaCl}$ the reduction of $\mathrm{HMF}$ varied from 18 to $61 \%$ [32].

Due to the numerous application possibilities and various methods that enable the most suitable solutions for the incorporation of certain functional ingredients into food matrix, microencapsulation will certainly find its place in the commercial production of flour-based confectionery products.

\section{Reference}

1. CAOBISCO Annual Report (2016). www.CAOBISCO.EU (10/05/2020).

2. Oručević Žuljević Sanja (2019) Trends in Flour Based Confectionary. Invited lecture presentation. $10^{\text {th }}$ International Congress Flour-Bread ' 19 and $12^{\text {th }}$ Croatian Congress of Cereal Technologists Brašno-Kruh '19. June, 11-14. Osijek, Croatia.

3. Nakov G, V Stamatovska, Lj Necinova, N Ivanova, S Damyanova, et al. (2016) Sensor analysis of functional biscuits. Ukrainian Food Journal 5(1): 56-62.

4. Khalil MM, MT Shalaby, AD El Dahsha, SK Saeed (2015) Chemical and Biological Studies on Functional Biscuits. J Food and Dairy Sci Mansoura Univ 6(5): 277-292.

5. Padma Ishwarya SP, Prabhasankar (2013) Fructooligosaccharide Retention during baking and its influence on biscuit quality. Food Bioscience 4: 68-80.

6. Butnariu M, I Sarac (2019) Functional Food. International Journal of Food Sciences and Nutrition. 3(3): 7-16.

7. Jain SC (2012) Potential of bakery products as functional foods. J Food Process Technol 3(10): 56.

8. Islamović A, M Mlačo, N Berbić, A Begić Akagić, S Orucević, et al. (2014) Seasonal variation of the physical and chemical parameters of wild genotypes of cornelian cherry (Cornus mas L.). Journal of International Scientific Publications: Agriculture and Food 2: 466-471.
9. Hegazy AK, SL Al Rowaily, M Faisal, AA Alatar, MI El Bana, et al. (2013) Nutritive value and antioxidant activity of some edible wild fruits in the Middle East. J Med Plant Res 7: 938-946.

10. Chakravarty S, KD Bhutia, CP Suresh G, Shukla NA, Pala NA, et al. (2016) A review on diversity, conservation, and nutrition of wild edible fruits. J Nat Appl Sci 8: 2346-2353.

11. Akagić A, A Vranac Oras, S Oručević Žuljević, N Spaho, P Drkenda, et al. (2020) Geographic Variability of Sugars and Organic Acids in Selected Wild Fruit Species. Foods 9(4): 462-476.

12. Lakkis JM Encapsulation and controlled release in bakery applications. In Encapsulation and Controlled Release Technologies in Food Systems (2016) EDS. Jamileh M, Lakkis, Second Edition 204-235.

13. Noshirvani N (2014) an overview of encapsulation technologies for food applications.

14. Kashappa G, H Desai, HJ Park (2005) Recent Developments in Microencapsulation of Food Ingredients. Drying Technology: An International Journal 23(7): 1361-1394.

15. Haffner FB, D Roudayna, A Pasc (2016) Encapsulation of probiotics: insights into academic and industrial approaches. AIMS Materials Science 3(1): 114-136.

16. Muzzafar A, V Sharma (2018) Microencapsulation of probiotics for incorporation in cream biscuits. Journal of Food Measurement and Characterization 12: 2193-2201.

17. Chawda PJJ, Shi S, Xue S, Y Quek (2017) Co-encapsulation of bioactives for food applications. Food Quality and Safety 1-8.

18. Jeyakumari A, AA Zynudheen, U Parvathy (2016) Microencapsulation of bioactive food ingredients and controlled release-a review. MOJ Food Process Technol 2(6): 214-224.

19. Tatar F, A Cengiz, T Kahyaoglu (2015) Evaluation of ultrasonic nozzle with spray drying as a novel method for the microencapsulation of blueberry's bioactive compounds. Innovative Food Science and Emerging Technologies 32: 136-145.

20. Vinceković M, M Viskić, S Jurić, J Giacometti, D Bursać Kovačević, et al .(2017) Innovative technologies for encapsulation of Mediterranean plants extracts. Trends in Food Science \& Technology 69: 1-12.

21. AlvimI ID, M Abreu Stein, I Paes Koury, FB Hellmeister Dantas, CL de Camargo Vianna Cruz, et al. (2016) Comparison between the spray drying and spray chilling microparticles contain ascorbic acid in a baked product application. LWT - Food Science and Technology 65: 689-694.

22. Shrestha AK, J Arcot, SDhital, S Crennan (2012) Effect of Biscuit Baking Conditions on the Stability of Microencapsulated 5-Methyltetrahydrofolic Acid and Their Physical Properties. Food and Nutrition Sciences (3): 1445-1452.

23. Wilson N, NP Shah (2007) Microencapsulation of Vitamins. ASEAN Food Journal 14(1): 11-14.

24. Kulthe AA, SS Thorat, SB Lande (2017) Preparation of $\beta$-Carotene Enriched Pearl Millet Based Cookies. International Journal of Current Microbiology and Applied Sciences 6(2): 1197-1203.

25. Luca A, B Cilek, S Sahin, V Hasirci, G Smnu, et al.(2014) Storage and Baking Stability of Encapsulated Sour Cherry Phenolic Compounds Prepared from Micro- and Nano-Suspensions. Food Bioprocess Technol 7: 204-211.

26. Dordoni RGD, Garrido L, Marinoni L,Torri M, Piochi G, et al. (2019) Enrichment of Whole Wheat Cocoa Biscuits with Encapsulated Grape Skin Extract. International Journal of Food Science p. 1-11.

27. Elsebaie EM, RY Essa (2018) Microencapsulation of red onion peel polyphenols fractions by freeze drying technicality and its application in cake 42(7): e13654. 
28. Papillo VAM, Locatelli F, Travaglia M, Bordiga C, Garino JD, et al. (2018) Cocoa hulls polyphenols stabilized by microencapsulation as functional ingredient for bakery applications. Food Research International 115: 511-518.

29. Rosha GAC, S Favaro Trindade, CRF Grosso (2012) Microencapsulation of lycopene by spray drying: Characterization, stability, and application of microcapsules. Food and Bioproducts Processing 90(1): 37-42.

30. Goncalves ND, F De Lima Pena, A Sartoratto, C Derlamelina, MCT, Duarte AEC, et al. (2017) Encapsulated thyme (Thymus vulgaris) essential oil used as a natural preservative in bakery product. Food Research International 96: 154-160.
31. Gómez Mascaraque LG, M Hernández Rojas, P Tarancón, M Tenon, amparo lopez-rubio, et al. (2017) Impact of microencapsulation within electrosprayed proteins on the formulation of green tea extract-enriched biscuits. LWT -Food Science and Technology 81: 77-86.

32. Fiore A, AD Troise BA Mogol, V Roullier, A Gourdon, et al. (2012) Controlling the Maillard Reaction by Reactant Encapsulation: Sodium Chloride in Cookies. Journal of Agricultural and Food Chemistry 60(43): 10808-10814. 